\begin{tabular}{|c|c|c|}
\hline Beitr. Ent. & Keltern & ISSN 0005-805X \\
\hline $\mathbf{5 6}(2006) 2$ & S. $269-279$ & 15.12 .2006 \\
\hline
\end{tabular}

\title{
Thecophora pusilla auct. - ein Artenkomplex
}

\section{(Diptera: Conopidae)}

Mit 12 Figuren

Jens-Hermann Stuke

\section{Zusammenfassung}

Für zwei unter Thecophora pusilla auct. vereinigte Arten werden die Namen Thecophora cinerascens Meigen, 1804 und Thecophora bimaculata (Preyssler, 1791) eingesetzt [status rev.]. Ein neues Synonym wird festgestellt [syn. nov.]: Thecophora pusilla (Meigen, 1824) = Thecophora cinerascens (Meigen, 1804). Ein Bestimmungsschlüssel für die europäischen Arten der Thecophora atra-Artengruppe wird vorgelegt.

\section{Summary}

Two species previously overlooked as Thecophora pusilla auct. are here identified as Thecophora cinerascens Meigen, 1804 and Thecophora bimaculata (Preyssler, 1791) [status rev.]. One new synonym is introduced [syn. nov.]: Thecophora pusilla (MeIgen, 1824) $=$ Thecophora cinerascens $($ MeIgen, 1804). A key to the European species of the Thecophora atra species-group is presented.

Stichwörter

Diptera, Conopidae, Thecophora, syn. nov., status rev., Bestimmungsschlüssel, Europa

Key words

Diptera, Conopidae, Thecophora, syn. nov., status rev., key, Europe

\section{Einleitung}

Thecophora pusilla (MeIgen, 1824) ist eine im weiblichen Geschlecht durch die Form der Theka charakterisierte Art. In anderen Merkmalen, wie der Bestäubung, der Beinfärbung oder der Größe schien diese Art sehr variabel zu sein. Bei der Untersuchung nearktischen Materials der Gattung Thecophora RondanI, 1845 konnte festgestellt werden, dass die Beinfärbung bei den dort getrennten Arten wenig variabel ist und als Unterscheidungsmerkmal herangezogen werden kann. Die anschließende Analyse des vorliegenden Materials von Thecophora pusilla zeigte, dass unter Thecophora pusilla auct. zwei Arten zusammengefasst werden. Diese beiden Arten werden nach einem diagnostischen Merkmal im Folgenden hell- und dunkelbeinige Form von Thecophora pusilla auct. genannt. In dieser Arbeit wird die Abgrenzung der zwei Arten voneinander und von ähnlichen Thecophora-Arten Europas dargestellt und die Nomenklatur diskutiert. 
Nomenklatur der beiden Formen von Thecophora pusilla auct.

Die Nomenklatur innerhalb der Gattung Thecophora ist für die europäischen Arten unbefriedigend geklärt. 28 nominelle europäische Arten sind bekannt (ChVÁla \& SMITH 1988, Stuke \& Clements 2005), die zu acht validen Arten gehören. Von den 20 aus Europa beschriebenen Synonymen wird Thecophora sundewalli (ZETTERSTEDT, 1844) Thecophora fulvipes (Robineau-Desvoidy, 1830) zugeordnet, alle anderen Arten werden als Synonyme zu Thecophora atra (FABRICIUS, 1775) gestellt. Diese Zuordnung geht auf Meigen (1824) und Schiner (1862) zurück. Sie muss aus heutiger Sicht als pragmatische Lösung verstanden werden, da die Originalbeschreibungen in der Regel keine Zuordnung zu den sehr ähnlichen Arten zulassen und das Typenmaterial der Autoren Preyssler, Meigen, Fabricius und Robineau-Desvoidy in der Regel zerstört oder verschollen ist. Nur drei von Robineau-Desvoidy beschriebene Arten konnten kürzlich von Stuke \& Clements (2005) untersucht werden: Thecophora femoralis (RobineauDesvoidy, 1853) und Thecophora meigeni (Robineau-Desvoidy, 1853) sind tatsächlich Synonyme von Thecophora atra; Thecophora nitidula (RoBIneAu-Desvoidy, 1853) ist ein Synonym von Zodion cinereum (Fabricius, 1794). Es ist allerdings unwahrscheinlich, dass Robineau-Desvoidy $(1830,1853)$, der 11 Synonyme von Thecophora atra beschrieben haben soll, keine der anderen in der Umgebung Paris nicht selten vorkommenden Thecophora-Arten vorliegen hatte.

Um geeignete Namen für die zwei unter Thecophora pusilla auct. zusammengefassten Taxa zu finden, werden die Synonyme von Thecophora atra geprüft und die beiden ältesten möglichen Namen eingesetzt. Der Name Thecophora pusilla (Meigen, 1824) sollte nach Möglichkeit nicht verwendet werden, damit in zukünftigen Arbeiten erkennbar ist, ob die zwei Formen von Thecophora pusilla auct. getrennt wurden oder nicht.

Die Synonyme der nicht zuzuordnenden oder jüngeren Namen werden nicht diskutiert, obwohl bei einigen Arten schon aus der Originalbeschreibung deutlich wird, dass die Synonymie mit Thecophora atra nicht zutreffen kann. Allerdings ist auch eine andere fundierte Zuordnung meist nicht möglich. Das von Chvíla \& SMith (1988) Thecophora pusilla zugeordnete Synonym Thecophora abdominalis (CHEN, 1939) wird in dieser Arbeit nicht berücksichtigt, da die Art aus China beschrieben ist und kein Material einer der beiden Formen aus China vorliegt und außerdem der Name der jüngste aus der Liste der Synonyme wäre.

\section{Conops bimaculata Preyssler, 1791: 133-136}

Die Dipterensammlung Preysslers und damit auch Typenmaterial von Conops bimaculata sind zerstört (Evenhuis 1997). Die Angabe aus der Originalbeschreibung von Conops bimaculata „Der Schenkel ist aus allen Theilen des Fusses der längste, an der Wurzel bleichgelb" schließt die dunkelbeinige Form von Thecophora pusilla auct. aus und entspricht der hellbeinigen Form. Da die sehr detaillierte Beschreibung ansonsten ebenfalls der hellbeinigen Form von Thecophora pusilla entspricht, wird der Name Thecophora bimaculata (Preyssler, 1791) eingesetzt [status rev.]. Es ist erfreulich, dass die außergewöhnlich präzise und ausführliche Beschreibung PreYsslers hiermit gewürdigt werden kann. 


\section{Myopa annulata FABRICIUS, 1794: 399}

Die Angabe aus der Originalbeschreibung "Pedes testacei femoribus tibiisque annulo nigro" schließt beide Formen von Thecophora pusilla auct. aus. Der Typus befindet sich im Zoologischen Museum der Universität Kopenhagen (ZMUC), nach Informationen von Thomas Pape ist dieser Typus genau wie der Typus von Thecophora atra von Dermestiden zerfressen und es befinden sich an der Nadel nur noch Reste von Muskelgewebe.

\section{Myopa cinerascens MEIGEN, 1804: 287}

Der Typus konnte in Paris nicht entdeckt werden und muss als verschollen gelten. Die Angabe in der Originalbeschreibung „die Füfse haben schwarze glänzende Schenkel, die hintern mit gelber Wurzel; Schienenbeine und Fufsglieder sind graulich mit weifsem Schiller" schließt die hellbeinige Form von Thecophora pusilla auct. aus. Da kein Widerspruch zu dem hier vorgelegten Konzept der dunkelbeinigen Form von Thecophora pusilla auct. besteht, wird für diese Art der Name Thecophora cinerascens (MeIgen, 1804) gebraucht [stat. rev.].

\section{Myopa maculata MeIGEN, 1804: 287-288}

Der Typus konnte in Paris nicht entdeckt werden und muss als verschollen gelten. Die Angabe „Die vordersten und mittlern Füfse sind ganz schwarzbraun, die Hinterfüfse haben gelbe Schenkel mit schwarzer Spizze, die Schienenbeine eine gelbe Wurzel“ schließt die hellbeinige Form von Thecophora pusilla auct. aus.

\section{Myopa micans MeIGEN, 1804: 288}

Der Typus konnte in Paris nicht entdeckt werden und muss als verschollen gelten. Die Angabe „Die Füfse sind rotbraun“ schließt beide Formen von Thecophora pusilla auct. aus.

\section{Myopa femorata FABRICIUS, 1805: 181-182}

Die Angabe „femoribus basi nigris“ schließt beide Formen von Thecophora pusilla auct. aus. Der Typus befindet sich im ZMUC und wurde nicht untersucht.

\section{Myopa pusilla MeIGeN, 1824: 150}

Der Typus konnte in Paris nicht entdeckt werden und muss als verschollen gelten. Die Zuordnung zur dunkelbeinigen Form von Thecophora pusilla auct. erfolgt aufgrund der Angabe „Beine glänzend schwärzlich; Hinterschenkel an der Wurzel und alle Knie rötlich." Thecophora pusilla (Meigen, 1824) muss als jüngeres Synonym zu Thecophora cinerascens (Meigen, 1804) gestellt werden [syn. nov.].

\section{Diagnose}

Beide Formen von Thecophora pusilla auct. gehören in eine Gruppe von schwer unterscheidbaren Arten, die sich (1) durch das Fehlen eines Paares submedialer schwarzer unbestäubter Streifen auf dem vorderen Teil des Mesoscutums, (2) ein bei den meisten Arten unregelmäßig grau bestäubtes Abdomen, (3) einen Pedicellus, der in der Regel länger ist als der Postpedicellus, (4) einem (gelegentlich undeutlich ausgebildeten) dunklen Fleck vorne im Mundinnenrand und (5) den an der Hinterseite höchstens schwach 
bestäubten $\mathrm{f} 1$ auszeichnen. Aus der so charakterisierten Thecophora atra-Artengruppe sind sechs Arten aus Europa nachgewiesen. Thecophora jakutica Zimina, 1974 ist erst kürzlich von KaHANpäÄ (in Vorbereitung) für Finnland und das angrenzende Russland und damit erstmals für Europa nachgewiesen worden. Während der Vorbereitungen dieser Arbeit konnte auch ein Beleg aus Mitteleuropa entdeckt werden (1 9 , Deutschland, Fichtelgebirge, Kulm $2 \mathrm{~km}$ S Weidenberg, 10.-24.VII.1993, leg. Wolff, coll. Stuke, Fig. 5). Da Thecophora jakutica sicherlich bisher übersehen wurde, ist ihre Verbreitung in Europa nicht zu beurteilen. Die ebenfalls in den Schlüssel mit aufgenommene Thecophora apivora ZIminA, 1968 ist noch nicht für Europa publiziert gewesen, es liegt aber ein unpublizierter Nachweise aus Aserbaidschan vor (1 9 , Azerbaijan S Baku, Altyagach, 1200 m, 4050'N, 4850'E, 22.VI.1996, leg. Hauser, coll. Stuke, Fig. 1).

Die Diagnose der Weibchen der sechs europäischen Arten ist im folgenden Bestimmungsschlüssel dargestellt. Die Unterscheidung der beiden Formen von Thecophora pusilla auct. - Thecophora cinerascens und Thecophora bimaculata - sind zusätzlich in Tabelle 1 gegenübergestellt.

Die Bestimmung der Männchen der sechs Arten ist nach meinem Kenntnisstand höchstens möglich, wenn gleichzeitig Weibchen aus einem Gebiet vorliegen und das Artenspektrum einer Region gut eingeschätzt werden kann. So lässt sich im nördlichen Mitteleuropa Thecophora bimaculata aufgrund der Beinfärbung von Thecophora atra und Thecophora cinerascens trennen. In Südeuropa kann aber auch Thecophora atra heller gefärbte Beine haben. In vielen Gegenden Europas ist die Kenntnis noch so lückenhaft, dass eine Bestimmung der Männchen nicht möglich ist.

\section{Bestimmungsschlüssel der Weibchen europäischer Arten der Thecophora atra- Artengruppe}

1 Mesoscutum an der Vorderseite mit einem Paar schmaler, scharf abgegrenzter schwarzer (unbestäubter) submedialer Streifen; Mundinnenrand in der Regel ohne dunklen Fleck an der Vorderseite; Pedicellus meist deutlich länger als Postpedicellus; Abdomen gleichmäßig grau oder golden bestäubt; f1 an der konvexen Hinterseite kräftig bestäubt, kräftiger als an der Vorderseite und auch kräftiger als bei $\mathrm{f} 2$ und $\mathrm{f} 3$.

Thecophora distincta - Artengruppe

- $\quad$ Mesoscutum mit einem schwarzen (unbestäubten) medialen Streifen oder ungestreift; Mundinnenrand in der Regel mit dunklem Fleck an der Vorderseite; Pedicellus etwa so lang wie Postpedicellus; Abdomen bei den meisten Arten mit unregelmäßiger grauer Bestäubung; f1 an der konvexen Hinterseite höchstens schwach bestäubt, weniger kräftig als an der Vorderseite und nicht stärker als bei $\mathrm{f} 2$ und f3. ....... (Thecophora atra - Artengruppe) 2

$2 \quad$ Theka apikal zugespitzt (Fig. 3, 6) . ...................................................................... 3

Theka apikal abgerundet (Fig. 1-2, 4-5, 7-12). ........................................................ 4

3 Abdomen und Thorax golden bestäubt, Tergit 2-5 dorsal oft gleichmäßig bestäubt [gelegentlich schwer erkennbares Merkmal]; Mesoscutum im vorderen Bereich mit zwei breiten, gut abgegrenzten submedialen Bestäubungsstreifen (in Aufsicht); f1 zumindest ventral orangebraun, $\mathrm{f} 2$ oft überwiegend gelb; Propleuron ventral typischerweise mit 1-3 langen kräftigen Borsten und mehreren kleiner Borsten; größere Art. 
- $\quad$ Abdomen und Thorax silbergrau bestäubt, Tergit 2-5 in der Regel am Hinterand stärker bestäubt; Bestäubungsstreifen auf Mesoscutum variabel, oft fehlend oder zu einem medialen, undeutlich abgegrenzten, breiten Bestäubungsstreifen verschmolzen; $\mathrm{f} 1$ bei mitteleuropäischen Tieren typischerweise vollständig schwarz, f2 höchstens basal schmal gelb [aus Südeuropa und Nordafrika liegen Tiere mit überwiegend gelben $\mathrm{f} 1$ und $\mathrm{f} 2 \mathrm{vor}$; Propleuron ventral typischerweise neben einer langen breiten Borsten mit 0-3 deutlich kleineren Borsten; kleinere Art. atra (FABRICIUs, 1775)

$4 \quad \mathrm{f} 1$ und $\mathrm{f} 2$ zumindest basal gelb, $\mathrm{f} 1$ oft ventral vollständig gelb; $\mathrm{f} 3$ mindestens in der basalen Hälfte gelb, oft nur die Spitze schwarz; Theka relativ schmal (Fig. 10-12).

bimaculata (PreYssLer, 1791)

f1 und f2 schwarz; f3 meist nur in der basalen Hälfte gelb; Theka breiter (Fig. 1-2, 4-5, 7-9).

5 Theka entsprechend Fig. 1-2: Borstenfeld sehr breit und mit 5 abgegrenzten Borstenreihen; Stirn einschließlich Ocellendreieck gelb bis orangebraun ohne deutlich schwarz abgegrenzten hinteren Bereich. apivora ZiminA, 1968

- Theka schmaler und mit weniger abgegrenzten Borstenreihen; Stirn je nach Art unterschiedlich.

6 Theka ragt nur wenig hervor (Fig. 4, 5), daher erreicht das Borstenfeld lateral fast das Ende der Tergite; Tergit 2 und 3 schmaler, länger als breit; Tergit 5 und 6 oft etwa gleich schmal, deutlich schmaler als Tergit 4; Stirn einschließlich Ocellendreieck gelbbraun; Behaarung auf der vorderen Hälfte des Mesoscutums kürzer. . jakutica Zimina, 1974

- Theka ragt deutlicher hervor (Fig. 7-9), das Borstenfeld ist vom Unterrand des Tergites deutlich getrennt; Tergit 2 und 3 meist kürzer, kürzer als breit; Tergit 5 oft deutlich schmaler als die etwa gleich langen Tergite 4 und 6; oberer Teil der Stirn einschließlich Ocellendreieck in der Regel schwarz, deutlich zum helleren vorderen Teil kontrastierend; Behaarung auf dem vorderen Teil des Mesoscutums länger. cinerascens (MEIGEN, 1804)

Tab. 1: Unterscheidung von Thecophora cinerascens (Meigen, 1804) und Thecophora bimaculata (Preyssler, 1791).

\begin{tabular}{|c|c|}
\hline $\begin{array}{l}\text { cinerascens }(\text { MeIgeN, 1804) } \\
=\text { pusilla }(\text { MeIgEN, 1824) }\end{array}$ & bimaculata (PreYssLer, 1791) \\
\hline $\begin{array}{l}\mathrm{f} 1+\mathrm{f} 2 \text { schwarz; } \mathrm{f} 3 \text { in der Regel höchstens in der basalen } \\
\text { Hälfte gelb }\end{array}$ & $\begin{array}{l}\mathrm{f} 1+\mathrm{f} 2 \text { zumindest an der Basis gelb, f1 häufig } \\
\text { ventral vollständig gelb; } \mathrm{f} 3 \text { zumindest in der ba- } \\
\text { salen Hälfte gelb, oft nur die Spitze dunkel }\end{array}$ \\
\hline of 오:Theka relativ breit (Fig. 7-9) & 우 ㅇ: Theka relativ schmal (Fig. 10-12) \\
\hline $\begin{array}{l}\text { Tergit } 6 \text { in der Regel nicht oder nur geringfügig medial } \\
\text { bestäubt }\end{array}$ & Tergit 6 medial deutlich bestäubt \\
\hline $\begin{array}{l}\text { Flügelbasis weniger ausgedehnt gelb gefärbt: Radius nur } \\
\text { bis vor der Verzweigung der Adern R1 und R } 2+3 \text { gelb, } \\
\text { danach schwarz }\end{array}$ & $\begin{array}{l}\text { Flügelbasis ausgedehnter gelb gefärbt: Radius } \\
\text { auch noch nach der Verzweigung der Adern R1 } \\
\text { und } R 2+3 \text { gelb }\end{array}$ \\
\hline
\end{tabular}



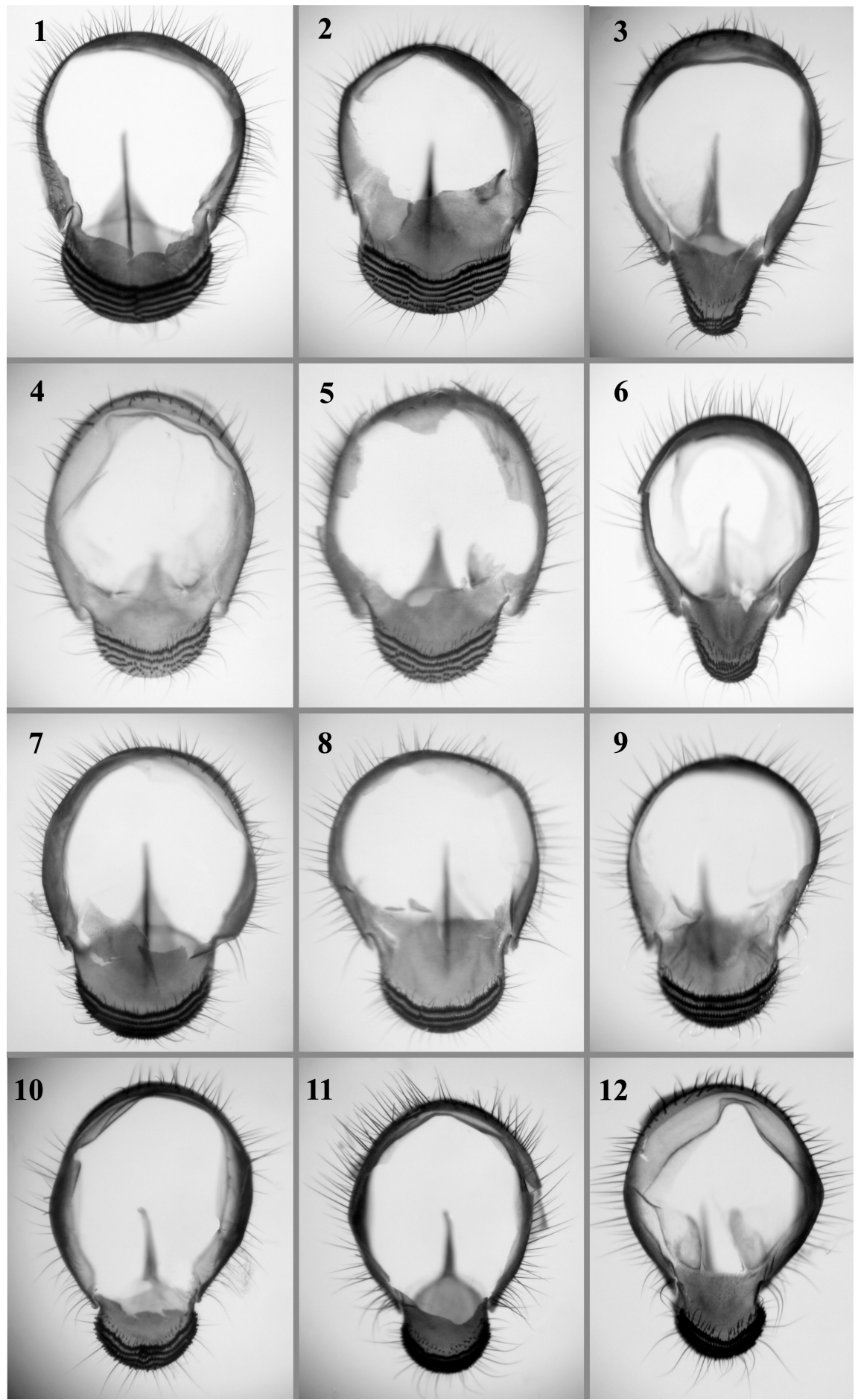


\section{Key to the females of European Species of the Thecophora atra species-group}

1 Mesoscutum in the front part with a pair of narrow, well-defined, undusted submedial stripes; interior edges of oral cavity normally without dark markings; pedicellus usually obviously longer than postpedicellus; abdomen evenly greyish or golden dusted; f1 strongly dusted on the convex posterior side, more strongly than on the anterior side and also more strongly than on $\mathrm{f} 2$ and $\mathrm{f} 3$. Thecophora distincta species-group

- Mesoscutum in the front part with only one median undusted stripe, or with none; interior edges of oral cavity normally with dark markings on either side at front; Pedicellus approximately as long as postpedicellus; abdomen in most specimens with uneven greyish dusting; f1 at most weakly dusted on the convex posterior side, less strongly dusted than on the anterior side, and less strongly than on $\mathrm{f} 2$ and $\mathrm{f} 3$.

Thecophora atra species-group - 2

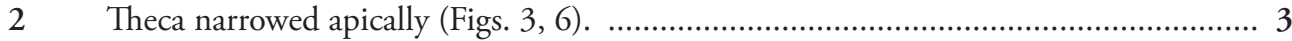

Theca rounded apically (Figs. 1-2, 4-5, 7-12). ................................................... 4

3 Abdomen and thorax golden dusted, tergites 2-5 usually evenly dusted dorsally [but this characteristic sometimes variable]; Mesoscutum in the anterior area usually with two broad, well-defined submedian dusted stripes (when viewed from above); f1 at least ventrally orange-brown, f2 often mainly yellow; Propleuron typically with 1-3 long, strong bristles ventrally, together with several smaller bristles; larger species.

fulvipes (Robineau-Desvoidy, 1830)

Abdomen and thorax silvery-grey dusted, tergites 2-5 normally more strongly dusted towards the posterior edge; Submedian dust stripe on mesoscutum variable, may be more-or-less absent, or more usually a single inconspicuous broad stripe, sometimes showing signs of narrow separation into two stripes; $\mathrm{f} 1$ in central European specimens is typically completely black, with $\mathrm{f} 2$ at most narrowly yellow at base [in southern Europe and North Africa there are specimens available with $\mathrm{f} 1$ and $\mathrm{f} 2$ mainly yellow]; propleuron typically with one long, strong bristle ventrally, together with $0-3$ obviously smaller bristles.

atra (FABRICIUS, 1775)

$4 \quad \mathrm{f} 1$ and $\mathrm{f} 2$ yellow at least basally, $\mathrm{f} 1$ often completely yellow ventrally; $\mathrm{f} 3$ at least in the basal half yellow, often with only the apex black; theca relatively narrow (Figs. 10-12).

bimaculata (Preyssler, 1791)

$\mathrm{f} 1$ and $\mathrm{f} 2$ black; f3 usually only yellow in the basal half; theca broader (Figs. 1-2, 4-5, 7-9).

Fig. 1-12: Theka (dargestellt ist das Syntergit 5) verschiedener Thecophora-Arten. - 1: apivora Zimina, 1968 [Aserbaidschan, Altyagach]; - 2: apivora Zimina, 1968 [Paratypus, Russland, Aksy-Dzhabagly Zapovednik, Kordon Dagbaza]; - 3: atra (FABRICiUs, 1775) [Frankreich, B. du Rhóne la Fabrique, Durance]; - 4: jakutica Zimina, 1974 [Finnland, Nurmis]; - 5: jakutica Zimina, 1974 [Deutschland, Fichtelgebirge]; - 6: fulvipes (Robineau-Desvoidy, 1830) [Deutschland, Baden-Württemberg, Werbach (Tauber)]; - 7: cinerascens (Meigen, 1804) [Bulgarien, Melnik near Petric]; - 8: cinerascens (Meigen, 1804) [Deutschland, Niedersachsen, E Sack]; - 9: cinerascens (Meigen, 1804) [Deutschland, Niedersachsen, E Sack]; - 10: bimaculata (Preyssler, 1791) Deutschland, Baden-Württemberg, 05.VIII.1992, E13]; - 11: bimaculata (PreyssLer, 1791) [Tschechien, Chomutov]; - 12: bimaculata (Preyssler, 1791) [Bulgarien, Vlas near Nessebar]. 
5 Theca as in Figs. 1-2: Bristle field on theca very broad, with 5 well-defined rows of bristles; frons including ocellar triangle yellow or orange-brown, without an obviously defined black area at rear.

apivora Zimina, 1968

- Theca narrower, with four or fewer well-defined bristle rows; Frons variable, depending on the species. 6

6 Theca projecting only slightly (Figs. 4-5), therefore the bristle field almost meets the lower edges of the tergite laterally; tergites 2 and 3 narrower, longer than wide; tergites 5 and 6 usually of similar length, both clearly narrower than tergite 4; frons including ocellar triangle tawny; hairs on the anterior half of the mesoscutum shorter.

jakutica ZiminA, 1974

- $\quad$ Theca more obviously projecting (Figs. 7-9), the bristle field clearly separated from the lower edge of the tergite at sides; tergites 2 and 3 usually shorter than wide; tergite 5 usually clearly narrower than tergites 4 and 6 , which are both similar in length; upper part of the frons, including the ocellar triangle, normally black, contrasting clearly with the paler anterior part; Hairs on the anterior half of the mesoscutum longer.

cinerascens (MEIGEN, 1804)

Tab. 1: Differentiation of Thecophora cinerascens (Meigen, 1804) and Thecophora bimaculata (Preyssler, 1791).

\begin{tabular}{|c|c|}
\hline $\begin{array}{l}\text { cinerascens (MEIgEN, 1804) } \\
=\text { pusilla }(\text { MeIgEN, 1824) }\end{array}$ & bimaculata (PreyssLer, 1791) \\
\hline $\begin{array}{l}\text { f1 + f2 black; f3 normally at most yellow in the basal } \\
\text { half }\end{array}$ & $\begin{array}{l}\mathrm{f} 1+\mathrm{f} 2 \text { at least yellow at the base, } \mathrm{f} 1 \text { often com- } \\
\text { pletely yellow ventrally; } \mathrm{f} 3 \text { at least yellow in the } \\
\text { basal half, often with only the dorsal surface } \\
\text { dark }\end{array}$ \\
\hline 우 ㅇ: Theca relatively broad (Figures 7-9) & of o : Theca relatively narrow (Figures 10-12) \\
\hline Tergite 6 usually not or only negligibly dusted medially & Tergite 6 obviously dusted medially \\
\hline $\begin{array}{l}\text { Wing base with yellow coloration less prolonged: Radius } \\
\text { only yellowed until just before the branching of veins } \\
\text { R } 1 \text { and } R 2+3 \text {, then black beyond }\end{array}$ & $\begin{array}{l}\text { Wing base with yellow coloration more exten- } \\
\text { sive: Radius yellow well after the branching of } \\
\text { veins } R 1 \text { and } R 2+3\end{array}$ \\
\hline
\end{tabular}

\section{Verbreitung von Thecophora cinerascens und Thecophora bimaculata}

Thecophora bimaculata ist in Südeuropa und im südlichen Mitteleuropa verbreitet. Durch Deutschland scheint eine Verbreitungsgrenze zu verlaufen - es liegen Nachweise aus Baden-Württemberg und Rheinland Pfalz sowie von zwei Ostdeutschen Wärmeinseln vor. Folgende Belege [falls nicht anders angegeben coll. Stuke] konnten untersucht werden:

Bulgarien: 1 ㅇ, Melnik, near Petric, $450 \mathrm{~m}$, Malaisefalle, 11.V.-12.VI.1998, leg. Achterberg, de Vries \& Atanassova; - 1 ㅇ, Sv. Vlas near Nesebar, 20 m, Malaisefalle, 01.-30.VI.1998, leg. Achterberg, de Vries \& Atanassova; - 1 ㅇ, Sv. Vlas near Nesebar, 20 m, Malaisefalle, 01.-31.VII.1998, leg. Achterberg, de Vries \& Atanassova; - Deutschland: 3 우, Baden-Württemberg, Freiburg, Güterbahnhof, 16.VII.1993, leg. Stuke; - 1 , Baden-Württemberg, Freiburg, Mooswald Lehen, 21.VIII.1993, leg. Stuke; - 3 9 , Baden- 
Württemberg, Freiburg, Schönberg, 21.VI.1992, leg. Stuke; - 4 우 ㅇ, Baden-Württemberg, Freiburg, Schönberg, 27.VII.1994, leg. Stuke; - 1 \%, Baden-Württemberg, Heilbronn, $5 \mathrm{~km} \mathrm{NO,} \mathrm{Gellmersbach,}$ Dätzberg, 11.VIII.1995, leg. Schmid-Egger, coll. Hauser; - 1 ㅇ, Baden-Württemberg, Heuchelberg, 2 km N Brackenheim, Mönchsberg, 02.VIII.1993, leg. Schmid-Egger, coll. Hauser; - 1 ㅇ, Baden-Württemberg, Kaiserstuhl, Schehlingen, 23.VII.1993, leg. Stuke; - 1 \% , Baden-Württemberg, Mühlacker, Lienzingen, Hamberg, 04.VII.1991, leg. Schmid-Egger; - 1 \%, Baden-Württemberg, Mühlacker, Mühlhausen, Enzschlinge, 11.VIII.1990, leg. Schmid-Egger, coll. Hauser; - 1 , Baden-Württemberg, Pforzheim, Niefern, Lattenwald, leg. Schmid-Egger; - 1 \%, Baden-Württemberg, Südbaden, Istein, Rhein, 03.VI.1997, leg. Schmid-Egger, coll. Hauser; - 1 \%, Baden-Württemberg, Südschwarzwald, 5 km N WT Tiengen, Schlüchttal 2 km NO Witznau, 01.VII.1994, leg. Schmid-Egger, coll. Hauser; - 1 \% , Baden-Württemberg, Weinstetten, 27.V.1990, leg. Stuke; - 1 \% , Rheinland-Pfalz, Nahe, Schloß-Böckelheim, 29.VI.1995, leg. Schmid-Egger, coll. Hauser; - 1 , Sachsen-Anhalt, Burgenlandkreis, NSG „Tote Täler“ 1 km W Großwilsdorf, 09.VIII.16.VIII.2002, leg. Pietsch; - 1 ㅇ, Thüringen, Kyffhäuser, Kosackenberg, 20.VI.2002, leg. Burger; - Frankreich: 2 우 옹 Frankreich, Elsass, Mittelwald Dessenheim, 23.VIII.1996, leg. Stuke; - 2 ㅇ 우, Frankreich, La RocheClermault, F. Indre-et-Loire, 29.VIII. 1995, leg. Cocquempot, coll. Hauser; - Georgien: 1 , Sagajero, $30 \mathrm{~km}$

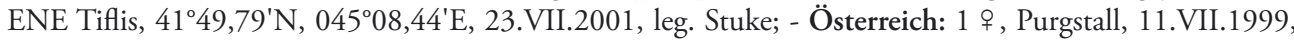
leg. Ressl; - Spanien: 1 ㅇ, El Burgo, 01.VII.1990, leg. T. Gijswijt; - Tschechien: 2 ㅇ ㅇ, Bohemia, Chrudim, Střemošická stráň, 02.VII.1997, leg. Mocek; - 2 ㅇ +, Bohemia, Chrudim, Střemošická stráń, 15.VIII.1997, leg. Mocek; - 1 † , Bohemia, Chrudim, Střemošická stráň, 160ㅗ'E, 4953'E, 01.VII.1998, leg. Mocek; - 1 \%, Moravia, Podyi, 1,5 km SW of Havraniky steppe, 340 m, 4848'29"N, 1559'31"E, 24.VII.-29.VIII.2001, leg. Barták \& Kubik; - 1 \&, Moravia, Podyi, Čížov-Hardecké stráně, steppe, 24.VIII.1995, leg. Mocek; - 1 ㅇ, Březno, Chomutov, Hutná brook, 285 m, 50²4'24"N, 13²3'21"E, 27.V.-18.VI.2000, leg, Barták; - 1 †, Březno, Chomutov, Hutná brook, 285 m, 50²4'24"N, 1323'21"E, 25.VIII.-30.IX.2000, leg, Barták; - Türkei: 2 ㅇ 우, Askale - Bayburt; 2400 m, 4001,92'N, 040³1,00'E, 20.VII.2005, leg. Stuke.

Thecophora cinerascens ist in Süd- und Mitteleuropa verbreitet, aus Skandinavien und England fehlen Nachweise. Thecophora cinerascens besiedelt eventuell kühlere Gebiete als Thecophora bimaculata - im nördlichen Mitteleuropa und in den Alpen konnte bisher nur Thecophora cinerascens nachgewiesen werden. Folgende Belege [falls nicht anders angegeben coll. Stuke] liegen vor:

Aserbaidschan: 1 ㅇ, Lerik, Zuvand, Gosmalian, $1300 \mathrm{~m}, 38^{\circ} 40^{\prime} \mathrm{N}, 48^{\circ} 20^{\prime} \mathrm{E}, 11 . \mathrm{VI} .1996$, leg. et coll. Hauser; - Bulgarien: 1 \% , Zelin near Botevgrad, 500 m, 09.V.-19.VI.1998, leg. Achterberg, de Vries \& Atanassova; - Deutschland: 2 우, Baden-Württemberg, Eppingen, Zaberfeld, Burghalde, 03.VIII.1993, leg. Windschnurer, coll. Hauser; - 1 \%, Baden-Württemberg, Freiburg, Schönberg, 21.VI.1992, leg. Stuke; - 1 ㅇ, Baden-Württemberg, Freiburg, Schönberg, 27.VII.1994, leg. Stuke; - 1 \%, Baden-Württemberg, Freiburg, Flughafen, 24.VII.1992, leg. Schmid-Egger; - 1 \%, Baden-Württemberg, Freiburg, Güterbahnhof, 12.VIII.1993, leg. Stuke; - 1 9 , Baden-Württemberg, Heilbronn, Gellmersbach, Dätzberg, 12.VIII.1990, leg. Schmid-Egger; - 1 \% , Baden-Württemberg, Heuchelberg, Brackenheim, 18.VI.1993, leg. Schmid-Egger, coll. Hauser; - 1 , Baden-Württemberg, Horb, Osterhalde, 30.VII.1991, leg. Schmid-Egger; - 3 $\uparrow$, Baden-Württemberg, Kaiserstuhl, Schehlingen, 23.VII.1993, leg. Stuke; - 1 \%, Baden-Württemberg, Kaiserstuhl, Schehlingen, 11.VIII.1993, leg. Stuke; - 1 \%, Baden-Württemberg, Kaiserstuhl, Schehlingen, 31.VIII.1993, leg. Stuke; - 1 ㅇ, Baden-Württemberg, Kieswerk Reiselfingen, 30.VII.1993, leg. Stuke; - 1 , Baden-Württemberg, Mühlacker, Lienzingen, Hamberg, 04.VII.1991, leg. Schmid-Egger; - 1 ㅇ, BadenWürttemberg, Mühlacker, Lomersheim, Hinter der Burg, 11.VII.1990, leg. Schmid-Egger, coll. Hauser; - 1 †, Baden-Württemberg, Mühlhausen, Kammerten, Berg, 18.VIII.1990, leg. Schmid-Egger, coll. Hauser; - 1 †, Baden-Württemberg, Müllheim, Exerzierplatz, 09.VI.1992, leg. Schmid-Egger; - 1 †, BadenWürttemberg, Müllheim, Exerzierplatz, 28.VII.1991, leg. Schmid-Egger; - 1 \%, Baden-Württemberg, Oberrheinebene, Ruine Keyserberg, 07.X.1993, leg. Stuke; - 1 , Baden-Württemberg, Pforzheim, Niefern, Lattenwald, 03.VIII.1990, leg. Schmid-Egger, coll. Hauser; - 1 , Baden-Württemberg, Pforzheim, Niefern, Lattenwald, 01.VII.1991, leg. Schmid-Egger; - 1 ㅇ, Baden-Württemberg, Pforzheim, Niefern, Lattenwald, 22.VI.1992, leg. Schmid-Egger, coll. Hauser; -2 ㅇ ㅇ , Baden-Württemberg, Schwäbische Alb, Beurener Heide, 
15.VIII.1993, leg. Stuke; - 1 † , Baden-Württemberg, Stromberg, 2 km E Diedenbach, Füllmenbach, Hofb., 02.VIII.1993, leg. Schmid-Egger, coll. Hauser; - 1 † , Baden-Württemberg, Stromberg, NW Hohenhaslach, Umgebung Teufelsberg, 02.VII.1993, leg. Schmid-Egger, coll. Hauser; - 1 , Baden-Württemberg, Stromberg, Weinberge E Leonbronn, 19.VI.1993, leg. Schmid-Egger, coll. Hauser; - 2 우, Baden-Württemberg, Stromberg, zwischen Sersheim und Hohenhaslach, 30.VI.1993, leg. Schmid-Egger, coll. Hauser; - 1 , Baden-Württemberg, Trockenaue Grisheim, 01.V.1993, leg. Stuke; - 2 ㅇ ․, Baden-Württemberg, Trockenaue Grisheim, 17.V.1997, leg. Stuke; - 1 \%, Baden-Württemberg, Vaihingen, Enz, Stromberg, Ochsenbach, 28.VII.1990, leg. Schmid-Egger, coll. Hauser; - 1 \% , Baden-Württemberg, Waldrohrbach, Forsthöhe, 23.V.1992, leg. Schmid-Egger, coll. Hauser; - 1 \% , Baden-Württemberg, Waldrohrbach, Forsthöhe, 08.VII.1993, leg. Schmid-Egger, coll. Hauser; - 2 우, Berlin, Schöneberg, Naturpark Südgelände, 05.VI.2002, leg. Saure; - 1 \% , Berlin, Adlershof, ehemaliger Sportplatz, Genossenschaftsstraße, 03.VI.2004, leg. Saure; - 1 q , Berlin, Kreuzberg, Görlitzer Park, ehemaliger Bahnhof, 16.VII.1998, leg. Esser; - 1 , Berlin, Lichterfelde, ehemaliger Truppenübungsplatz Lichterfelde Süd, 15.V.2000, leg. Saure; - 1 †, Berlin, Mitte, 10.VIII.2003, leg. Esser; - 1 + , Berlin, Treptow, Johannisthal, ehemaliger Mauerstreifen, 25.VI.1999, leg. Esser; - 1 ㅇ, Brandenburg, $10 \mathrm{~km} \mathrm{~N}$ Eberswalde, Serwest, Kernberg, (Schorfheide), 28.V.1994, leg. Schmid-Egger, coll. Stuke; -2 우, Brandenburg, Eberswalde, Klein Zieth, Serwester See, 13.VII.1994, leg. DEI, coll. Hauser; - 1 ㅇ, Hessen, Düne Griesheim, 29.VII.1994, leg. Stuke; - 1 ㅇ, Hessen, Griesheimer Sand, 23.VII.1992, leg. et coll. Hauser; - 1 ㅇ, Hessen, ND Rotböhl Sand, 29.VII.1994, leg. Stuke; - 2 우, Hessen, NSG-Griesheimer Düne, 09.VIII.1994, leg. et coll. Hauser; - 9 \% $~+$, Niedersachsen, Göttingen, Trockenrasen an „Emme“ W Dramfeld, 17.VIII.2005, leg. Stuke; - 7 ㅇ 9 , Niedersachsen, Göttingen, Trockenrasen E Sack, 16.VIII. 2005, leg. Stuke; - 3 ㅇ 우, Niedersachsen, Göttingen, Trockenrasen W Harste, 17.VIII.2005, leg. Stuke; - 2 ㅇ ㅇ, Niedersachsen, Göttingen, Trockenrasen W Groß Lengden, 17.VIII.2005, leg. Stuke; -5 우 우, Niedersachsen, Helmstedt, Heeseberg, 07.VII.2000, leg. Stuke; - 3 우 ㅇ, Niedersachsen, Helmstedt, Heeseberg, 15.VI.2003, leg. Stuke; - 1 ㅇ, Niedersachsen, Hildesheim, Trockenhänge Himmelberg NW Langenholzen, 26.V.2005, leg. Stuke; - 8 ㅇ 우, Niedersachsen, Hildesheim, Trockenhänge Himmelberg NW Langenholzen, 16.VIII.2005, leg. Stuke; - 1 \% , Niedersachsen, Langenholzen, Rehberg, 26.V.2005, leg. Stuke; - 3 우, Niedersachsen, Northeim, NSG „Altendorfer Berg“, 16.VIII.2005, leg. Stuke; - 3 ㅇ + , Niedersachsen, Northeim, NSG „Tönniesberg“ 1 km W Nienhagen, 16.VIII.2005, leg. Stuke; - 1 \% , Rheinland-Pfalz, Grünstadt, 1 km W Asselheim, Höllenberg, 150-220 m, 04.VIII.1993, leg. Schmid-Egger, coll. Hauser; - 1 \% , Rheinland-Pfalz, Grünstadt, Neuleiningen, Gemeindeberg, 08.VII.1993, leg. Schmid-Egger, coll. Hauser; - 1 , Rheinland-Pfalz, Bad Kreuznach, Oberhausen, Nahe, Bahndamm, 29.VI.1993, leg. Schmid-Egger, coll. Hauser; - 1 , Rheinland-Pfalz, Grünstadt, Neuleiningen, Gemeindeb., 12.VII.1994, leg. Schmid-Egger, coll. Hauser; - 1 \%, Rheinland-Pfalz, Ingelheim, Rheindamm, 18.VI.1992, leg. et coll. Hauser; - 2 ㅇ ㅇ, Rheinland-Pfalz, Ingelheim, Rheindamm, 15.VII.1992, leg. et coll. Hauser; - 1 \% , Rheinland-Pfalz, Landau, Waldrohrbach, Forsthöhe, 23.VI.1992, leg. Schmid-Egger; - 1 ㅇ, Rheinland-Pfalz, Mainz, Mombach, Sandäcker, an Waldfriedhof, 20.VII.1992, leg. Schmid-Egger; - 1 9, Rheinland-Pfalz, Umgebung Grünstadt, Dirmstein, 21.IX.1994, leg. Schmid-Egger, coll. Hauser; - 1 \%, Rheinland-Pfalz, Waldrohrbach, S Annweiler, Forsthöhe, 12.VII.1994, leg. Schmid-Egger, coll. Hauser; - 1 ㅇ, Sachsen-Anhalt, Burgscheidungen bei Freyburg / Unstrut, 25.VI.2000, leg. Esser; - 1 , Thüringen, Erfurt, Tongruben am Roten Berg, 28.VI.2000, leg. Burger; - Frankreich: 1 웅, Ury, F. Seine-etMan, 04.IX.1982, leg. Cocquempot, coll. Hauser; - Griechenland: 1 ㅇ, Vevi, 25.VIII.1983, leg. de Vrijer; - Italien: 1 ㅇ, Vindschgau, Kastelbell, 800 m, 21.VIII.1992, leg. et coll. Hauser; - 1 ㅇ, Valle d'Aosta, 10 km W Aosta, Fossaz, 900 m, 08.VII.1995, leg. Schmid-Egger, coll. Hauser; - 1 \%, Valle d'Aosta, 3 km W Aosta, Sarre, 800-1000 m, 06.VII.1995, leg. Schmid-Egger, coll. Hauser; - Kirgisien: 1 † , Amanbayevo N Talassky, Mt. r., 42.7N, 71.5 E, 13.VI.1986, leg. Milko; - Österreich: 1 ㅇ, Salzburg, 20.IX.1983, leg. Babiy; - 1 , Purgstall, 09.IX.1997, leg. Ressl, coll. Hauser; - 1 ㅇ, Purgstall, 17.IX.1997, leg. Ressl, coll. Hauser; - 1 , Purgstall, 06.VI.2000, leg. Ressl; - Spanien: 1 + , Andalusien, Sierra Filabres, 9 km E Albánchez, 23.IV.2003, leg. Halada; - Tschechien: 2 ㅇ , Bohemia, Polabská níž Vysoká nad Labem, 16.VI.1995, leg. Mocek; - 1 \%, Moravia, Podyi, 1,5 km SW of Havraniky steppe, 340 m, 4848'29"N, 1559'31"E, 24.VI.-21.VII.2001, leg. Barták \& Kubik; - 1 , Moravia, Podyij, Lišči akála, 2,5 km S Podmoli, 420 m, 4849'48"N, 1556'28"E, 24.-26.VI.2001, leg. Barták \& Kubik; - Türkei: 1 ㅇ, Askale - Bayburt; 1900 m, 4003,30'N, 040²7,10'E, 
20.VII.2005, leg. Stuke; - 1 9 , Erurum - Askale; 1700 m, 3956,64'N, 04045,80'E, 21.VII.2005, leg. Stuke; - 2 우 우, Erzurum, Atatürk University, 39 $9^{\circ} 54,11^{\prime} \mathrm{N}, 041^{\circ} 13,59^{\prime} \mathrm{E}, 19 . \mathrm{VII} .2005$, leg. Stuke; - 1 ㅇ, Erzurum Tortum, $1860 \mathrm{~m}, 40^{\circ} 15,63^{\prime} \mathrm{N}, 041^{\circ} 32,78^{\prime} \mathrm{E}, 26 . V I I .2005$, leg. Stuke; - 2 ㅇ 우, Horasan - Sarikamis, $1950 \mathrm{~m}$, $40^{\circ} 14,27^{\prime} \mathrm{N}, 042^{\circ} 38,84^{\prime} \mathrm{E}, 24$.VII.2005, leg. Stuke; - 1 ㅇ, Köprüköy - Karayazi, 2350 m, 3946,46'N, 042 01,60'E, 23.VII.2005, leg. Stuke; - Ungarn: 1 \% , Valencer See, Pakozd, 12.VIII.1991, leg. et coll. Hauser.

\section{Danksagung}

Martin Bode (Leer) half bei der Übersetzung lateinischer Originalbeschreibungen. Dr. Gunilla Ståhls (Helsinki) und Juha Laiho (Helsinki) ermöglichten die Bearbeitung von Thecophora jakutica. David Clements (Cardiff), Martin Hauser (Sacramento), und Danny Wolff (Ebstorf) stellten Material seltener Arten zur Verfügung. Thomas Pape (Kopenhagen) informierte mich über die Typen von Fabricius in der Sammlung des Zoologischen Museum der Universität Kopenhagen (ZMUC). Jere Kahanpää (Helsinki) stellte ein Manuskript zum Vorkommen von Thecophora jakutica in Finnland zur Verfügung. David Clements (Cardiff) half bei den englischen Übersetzungen. Wertvolle Hinweise zu früheren Manuskriptversionen verdanke ich Martin Hauser.

\section{Literatur}

Chvála, M. \& Smith, K. G. V. 1988: Conopidae. - In: Sóos, A. \& Papp, L. (Hrsg.), Catalogue of Palaearctic Diptera. Volume 8. Elsevier, Amsterdam-Oxford-New York-Tokyo: 363 S.

Evenhuis, N. L. 1997: Litteratura Taxonomica Dipterorum. - Leiden, Backhuys Publishers: 871 S.

Kahanpä̈̈, J. (in Vorbereitung): A review of Finnish Thecophora (Diptera: Conopidae). - Entomologica Fennica, Helsinki.

Meigen, J. W. 1824: Systematische Beschreibung der bekannten europäischen zweiflügeligen Insekten. 4. Theil. - Schultz Wundermann, Hamm: 416 S. + 11 Tafeln.

Robineau-Desvoidy, A. J. B. 1830: Essai sur les Myodaires. - Mémoires présentés par divers savants à l' académie royale des sciences de l' institut de france et imprimés par son ordre [sciences mathematiques et physiques], Paris, 2: 1-813.

Robineau-Desvoidy, A. J. B. 1853: Diptères des environs de Paris (1). Famille des Myopaires. - Bulletin de la société des sciences historiques et naturelles de Lyonne 7: 83-160.

Schiner, J. R. 1862: Fauna Austriaca. Die Fliegen (Diptera). Erster Theil. - Verlag von Carl Gerold's Sohn, Wien: 674 S. + 2 Tafeln.

Stuke, J.-H. \& Clements, D. 2005: The interpretation of some Conopidae (Diptera) described by Robineau-Desvoidy. - Zootaxa, Auckland 886: 1-12.

\section{Anschrift des Verfassers:}

Dr. Jens-Hermann Stuke

Brunnenstrasse 28

D - 26789 Leer

jstuke@zfn.uni-bremen.de

\section{Subject editor:}

F. Menzel 